\title{
Engineering Vibrio fischeri for Inducible Gene Expression
}

\author{
Jakob M. Ondrey and Karen L. Visick ${ }^{*}$
}

Department of Microbiology and Immunology, Health Sciences Division, Loyola University Chicago, 2160 S. First Avenue Building 105 Room 3936, Maywood, IL 60153, USA

\begin{abstract}
The marine bacterium Vibrio fischeri serves as a model organism for a variety of natural phenomena, including symbiotic host colonization. The ease with which the $V$. fischeri genome can be manipulated contributes greatly to our ability to identify the factors involved in these phenomena. Here, we have adapted genetic tools for use in $V$. fischeri to promote our ability to conditionally control the expression of genes of interest. Specifically, we modified the commonly used mini-Tn5 transposon to contain an outward-facing, LacI-repressible/IPTG-inducible promoter, and inserted the lacI gene into the $V$. fischeri chromosome. Used together, these tools permit the identification and induction of genes that control specific phenotypes. To validate this approach, we identified IPTG-controllable motility mutants. We anticipate that the ability to randomly insert an inducible promoter into the genome of $V$. fischeri will advance our understanding of various aspects of the physiology of this microbe.
\end{abstract}

Keywords: Genetics, inducible gene expression, motility, mutagenesis, Vibrio fischeri.

\section{INTRODUCTION}

Vibrio fischeri is a marine bacterium used as a model organism for studies of bioluminescence [1, 2], quorum sensing [3], biofilm formation [4, 5], and symbiosis [6, 7]. The discovery and characterization of these natural phenomena have been greatly facilitated by the development and use of genetic tools that disrupt and modify genes within the $V$. fischeri genome.

While the bioluminescent nature of $V$. fischeri was first described in the $19^{\text {th }}$ century by Martinus Willem Biejerinck [8], it was not until 1992 when the first genetic manipulation was described by Dunlap and Kuo [9]. These researchers successfully introduced foreign DNA via conjugation and generated gene replacement mutants via homologous recombination. Specifically, they manipulated a plasmidborne copy of the $V$. fischeri luminescence (lux) genes in Escherichia coli to obtain insertion and deletion mutants, then transferred the resulting constructs into $V$. fischeri, selecting for lux mutant recombinants. In 1994, the technique of transposon mutagenesis was applied to $V$. fischeri [10], thus permitting the investigation of uncharacterized genes and their involvement in phenotypes such as host colonization.

In subsequent years, the ability to manipulate $V$. fischeri genetically was facilitated by the identification of antibiotic resistance cassettes that functioned in this organism and, in concert, the construction and/or use of a variety of plasmids: those that were more stable were used for complementation,

*Address correspondence to this author at the Department of Microbiology and Immunology, Health Sciences Division, Loyola University Chicago, 2160 S. First Avenue Building 105 Room 3936, Maywood, IL 60153, USA; Tel: (708)216-0869; Fax: (708)216-9574; E-mail: kvisick@luc.edu while those that were less stable were used for recombination [11]. Importantly, the identification of a $V$. fischeri-specific origin of replication facilitated the construction of highly stable vectors that (1) could be maintained without antibiotic selection and (2) were compatible with other commonly used plasmids [12], allowing experiments that depended on the use of two plasmids [13]. In addition, constructs used for inserting genes in single copy at the $\operatorname{Tn} 7$ site of other bacteria [14] were successfully adapted for $V$. fischeri $[11,15]$, permitting single copy gene expression and complementation in the absence of antibiotic selection and making it possible to conduct well-controlled gain of function experiments in the context of animal colonization [16-19].

The whole genome of $V$. fischeri was sequenced and annotated in 2005 [20], allowing researchers to more readily utilize reverse genetics approaches. Facilitating such approaches, a counter-selectable suicide vector used to generate unmarked gene replacements in Vibrio splendidus [21] was adapted for use in V. fischeri. [17, 22]. This approach has permitted the reliable and rapid construction of unmarked mutations and the ability to readily generate strains with multiple unmarked mutations.

Although $V$. fischeri genetics has advanced greatly since 1992, some aspects remain under-developed. For example, little work has been done to develop inducible control over the expression of genes present in the chromosome. Although plasmid libraries have been developed [23, 24] and can be used to identify positive regulators of a given phenotype, such libraries are limited in the size of the insert and are unlikely to contain large operons intact. Here, we discuss the adaptation and use of a genetic tool for $V$. fischeri that allows for gene disruption as well as the insertion of a conditional promoter randomly into the genome, and can 
thus facilitate the identification of genes, such as positive regulators or large operons, whose induction drives a desired phenotype.

\section{MATERIALS AND METHODS}

\section{Strains and media}

The $V$. fischeri strains and plasmids constructed and/or used in this study are listed in Table 1. V. fischeri strain ES114 was used as the wild-type strain in these studies. $V$. fischeri strains were grown in complex LB-salt (LBS) medium [25] and tryptone broth-seawater (TB-SW) motility medium [26]. We also used the E. coli strains GT115 (Invivogen, San Diego, CA), CC118 [27], and $\pi 3813$ [21] for the purposes of cloning and conjugation. E. coli strains were grown using either LB [28] or Brain-heart infusion (BHI) (Difco) medium. The following antibiotics were added, as appropriate, at the indicated final concentrations: chloramphenicol $(\mathrm{Cm})$ at 1 to $5 \mu \mathrm{g} / \mathrm{ml}$ for $V$. fischeri and 25 $\mu \mathrm{g} / \mathrm{ml}$ for E. coli; tetracycline (Tc) at $5 \mu \mathrm{g} / \mathrm{ml}$ for $V$. fischeri and $15 \mu \mathrm{g} / \mathrm{ml}$ for E. coli; kanamycin (Kan) at $50 \mu \mathrm{g} / \mathrm{ml}$; erythromycin (Erm) at $5 \mu \mathrm{g} / \mathrm{ml}$ for V. fischeri and $150 \mu \mathrm{g} / \mathrm{ml}$ for E. coli; and ampicillin (Ap) at $100 \mu \mathrm{g} / \mathrm{ml}$. Thymidine was added to a final concentration of $300 \mu \mathrm{M}$ for the growth of $\pi 3813$ E. coli cells. Agar was added at a final concentration of $1.5 \%$ for solid media and $0.225 \%$ for the motility medium.

Plasmid and strain construction. Standard molecular biology, transformation, and conjugation approaches were used to generate the plasmids and strains used in this study. The primers used are shown in Table 2. Plasmid pJMO14 was used to insert the $\operatorname{lacl}^{q}$ gene into the chromosome between yeiR and $g \operatorname{lm} S$, adjacent to the attTn7 site (Fig. 2). To generate pJMO14, upstream and downstream sequences $(\sim 500$ bp) flanking the target insertion site were first amplified from the ES114 chromosome by PCR using primers 1484 and 1485 and primers 1486 and 1487, then joined using overlap extension PCR [22, 29]. The resulting DNA fragment, which consisted of the flanking sequences joined by non-native sequences including a NotI site, was ligated into suicide vector pKV363 to generate pJMO8. Finally, the $l a c I^{q}$ gene, which was amplified using PCR with pCA24N as a template and primers 1544 and1489, was cloned into the PCR cloning vector pJET1.2, then sub-cloned into NotI-digested pJMO8 to generate pJMO14. To insert lacl $I^{\mathrm{q}}$ into the chromosome, the method of Le Roux et al. [21] was used as described previously [22]. The insertion of $l a c I^{9}$ into ES114, generating KV6576, was confirmed using PCR with primers 974 and 975.

Plasmid pJMO10, which was used to deliver the Tn5+ promoter $(\mathrm{Tn} 5 \mathrm{P})$ for transposon mutagenesis, was constructed as follows. Oligonucleotides 1439 and 1440, which contained sequences for the LacI-repressible $\mathrm{P}_{\mathrm{A} 1 / 34}$ promoter [1, 30], were annealed and ligated into the ApaI/SpeI-digested Tn5 delivery plasmid pEVS170 [31]. The insertion of the promoter into Tn 5 was confirmed by sequencing.

Table 1. Bacterial strains and plasmids.

\begin{tabular}{|c|c|c|}
\hline Strain & Genotype & Reference \\
\hline \multicolumn{3}{|l|}{ V. fischeri } \\
\hline KV6576 & IG $\left(\right.$ yeiR-glmS)::lacI $I^{q}$ & This Study \\
\hline KV7432 & IG $($ yeiR-glmS $):: l a c I^{q}$ IG $\left(V F \_A 0340-V F \_A 0341\right):: \operatorname{Tn} 5 \mathrm{P}\left(\mathrm{Erm}^{\mathrm{r}}\right)$ & This Study \\
\hline Plasmid & Description & Reference \\
\hline pCA24N & $\operatorname{lacl}^{q}\left(\mathrm{Cm}^{\mathrm{r}}\right)$ & [43] \\
\hline pCLD46 & LacI-repressible RscS overexpression plasmid $\left(\mathrm{Cm}^{\mathrm{r}}\right)$ & {$[13]$} \\
\hline pJET1.2 & Commercial cloning vector $\left(\mathrm{Amp}^{\mathrm{r}}\right)$ & Fermentas \\
\hline pJMO8 & pKV363 containing the yeiR-glmS IG region $\left(\mathrm{Cm}^{\mathrm{r}}\right)$ & This Study \\
\hline pJMO10 & pEVS170 containing a promoter within the $\operatorname{Tn}(\operatorname{Tn} 5 \mathrm{P})\left(\operatorname{Kan}^{\mathrm{r}} \mathrm{Erm}^{\mathrm{r}}\right)$ & This Study \\
\hline pJMO14 & pJMO8 containing the $l a c I^{q}$ allele $\left(\mathrm{Cm}^{\mathrm{r}}\right)$ & This Study \\
\hline pKV363 & Mobilizable suicide vector $\left(\mathrm{Cm}^{\mathrm{r}}\right)$ & [22] \\
\hline pVSV105 & Mobilizable vector $\left(\mathrm{Cm}^{\mathrm{r}}\right)$ & {$[12]$} \\
\hline
\end{tabular}


Table 2. Oligonucleotides.

\begin{tabular}{|l|l|l|}
\hline \multicolumn{1}{|c|}{ Primer \# } & \multicolumn{1}{|c|}{ Name } & \multicolumn{1}{c|}{ Sequence } \\
\hline \hline 908 & 170Ext2 & GCACTGAGAAGCCCTTAGAGCC \\
\hline 974 & Tn7_primerdma89 & GCTAAAGCGGTGACGGTGGAGTAG \\
\hline 975 & Tn7_primerdma90 & CCTCACCCCAGATGGTTTGGCA \\
\hline 1439 & Tn5-P_out Spe/Apa-F & $\begin{array}{l}\text { CTAGTTTATCAAAAAGAGTGTTGACTTGTGAGCGGATAACAATGATACTTA } \\
\text { GATTCAATTGTGAGCGGATAACAATTTCACACAGGGCC }\end{array}$ \\
\hline 1440 & Tn5-P_out Spe/Apa-R & $\begin{array}{l}\text { CTGTGTGAAATTGTTATCCGCTCACAATTGAATCTAAGTATCATTGTTATC } \\
\text { CGCTCACAAGTCAACACTCTTTTTGATAAA }\end{array}$ \\
\hline 1484 & VF2371 F & CTTGATTTATACAGCGAAGG \\
\hline 1485 & VF2371 Not I R & TAGGCGGCCGCACTTAGTATGGTTTTGAAGAGTAATTAATGTTTATTG \\
\hline 1486 & VF2372 Not I F & CATACTAAGTGCGGCCGCCTATATTGTCTCTCTTAGAACAATTATTC \\
\hline 1487 & VF2372 R & GGTCGTGGGGAGTTTTATCC \\
\hline 1489 & LacINotI R & GCGGCCGCCGCTCACTGCCCGCTTTCC \\
\hline 1544 & LacIqNotI F & GCGGCCGCGACACCATCGAATGGTGCAAAAC \\
\hline
\end{tabular}

Wrinkled colony formation. Strains were grown overnight in LBS containing $\mathrm{CM}$ at $28^{\circ} \mathrm{C}$ with agitation, then subcultured into fresh medium and grown until they reached exponential phase. Cultures were then diluted to an optical density at $600 \mathrm{~nm}\left(\mathrm{OD}_{600}\right)$ of 0.2 , concentrated by centrifugation, and re-suspended in fresh medium. $10 \mu \mathrm{l}$ aliquots of each culture were spotted onto an agar plate and incubated at room temperature. Images of the wrinkled spots were captured at the indicated times.

Tn5P mutagenesis and identification of IPTGinducible motility mutants. Parental strain KV6576 was mutagenized with $\mathrm{Tn} 5 \mathrm{P}$ by performing a tri-parental conjugation [32] with two E. coli strains, one carrying pJMO10 and other carrying pEVS104 [32] and selecting for the insertion of the transposon using Erm-containing LBS (LBS-erm) plates. Resulting colonies were inoculated into TB-SW motility plates lacking or containing $1.75 \mathrm{mM}$ IPTG. Putative motility mutants with IPTG-controlled phenotypes were purified from motility plates by streaking onto LBSerm plates. These strains were then evaluated a second time to verify their motility phenotypes.

Identification of Tn5P insertion sites. To determine the site of the Tn5P insertion in the motility mutants, chromosomal DNA was isolated and digested using the HhaI restriction enzyme as previously described [31]. Digested DNA was then self-ligated and transformed into CC118 cells, and clones were selected on Erm-containing BHI agar. The resulting plasmids were sequenced using primer 908 .

Motility Assays. Motility mutants were grown overnight in LBS and the cultures were diluted to an $\mathrm{OD}_{600}$ of 0.4 . Cells were pelleted, washed, and re-suspended in LBS and $5 \mu 1$ aliquots were inoculated on the surface of TB-SW motility plates that contained the indicated amounts of IPTG. Representative images were captured and the diameters of the outer ring of the swimming cells were measured using a ruler.

\section{RESULTS AND DISCUSSION}

Generation of Tn5P, a Tn5 transposon with an outward-facing promoter. One genetic tool that remained to be developed for $V$. fischeri was a method for randomly inserting an inducible promoter into the genome. To develop this tool, we chose to use the LacI-repressible promoter A1/34 [1]. This promoter contains two LacI binding sites, one between the -35 and -10 sites and another that overlaps the transcriptional start site (Fig. 1), and has been shown previously to function as a strong promoter in $V$. fischeri when it was inserted directly upstream of an operon [1]. We then engineered the mini-Tn5 delivery vector pEVS170 [31] to contain this promoter within the transposable element in an outward-facing position; the insertion of the promoter was confirmed by sequencing. This transposon will be referred to as $\operatorname{Tn} 5 \mathrm{P}$, for $\mathrm{Tn} 5$ plus Promoter.

Insertion of the lacI gene into $V$. fischeri and phenotypic assessment. $V$. fischeri does not contain the lac operon or the lacI repressor gene. Therefore, to control expression from the promoter within $\mathrm{Tn} 5 \mathrm{P}$, it was necessary to introduce the lacI gene into $V$. fischeri. One location traditionally used to insert genes within the $V$. fischeri genome is the $\mathrm{Tn} 7$ site, which is positioned between yeiR and $\operatorname{glm} S$ (Fig. 2) [15, 33]. However, because this site is heavily utilized for single copy complementation (e.g., [34]), we chose to leave this site intact for future manipulations. Instead, we targeted the insertion of $\operatorname{lacl}^{q}$, a more highly transcribed allele of the lacI gene, to a region immediately adjacent to the $\operatorname{Tn} 7$ site (between $y e i R$ and the $\operatorname{Tn} 7$ site) (Fig. 2). The result was a strain, KV6576, that contains $l a c I^{q}$, retains an intact $\operatorname{Tn} 7$ site, and remains unmarked, allowing for the use of an antibiotic resistance marker in future manipulations.

With the generation of the lacl ${ }^{\mathrm{q}}$-containing $V$. fischeri, two questions arose: (i) Is the lacl $I^{\mathrm{q}}$ allele functional (e.g., does it control gene expression in V. fischeri?); and (ii) is the 


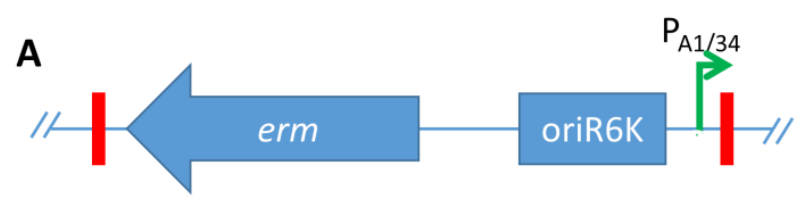

B

$$
\mathrm{P}_{\mathrm{A} 1 / 34} \text { Promoter }
$$

$-10$

$+1$

CTAGTTTATCAAAAAGAGTGTTGACTTGTGAGCGGATAACAATGATACTTAGATTCAATTGTGAGCGGATAACAAT

\section{TTCACACAGGGCCCTGTAAAACGACGGCCAGTCCTAGAGCGGCCGCAGATGTGTATAAGAGACAG}

Fig. (1). Construction of the Tn5P transposon. A) The Tn5 from pEVS170 contains an erythromycin resistance gene and an origin of replication (blue arrow and box) within the Tn5 ends (red rectangles). pEVS170 was modified to contain an outward-facing, LacIrepressible/IPTG-inducible promoter, $\mathrm{P}_{\mathrm{A} 1 / 34}$ (green arrow) [1]. (B) The $\mathrm{P}_{\mathrm{A} 1 / 34}$ promoter region (italics) contains two LacI binding sites (bold). The binding of LacI to these sites hinders access to the -35 and -10 sites (green), thus repressing transcription. The Tn 5 end (red) contains a start codon (underlined).

ES114

\section{Tn7 Site}

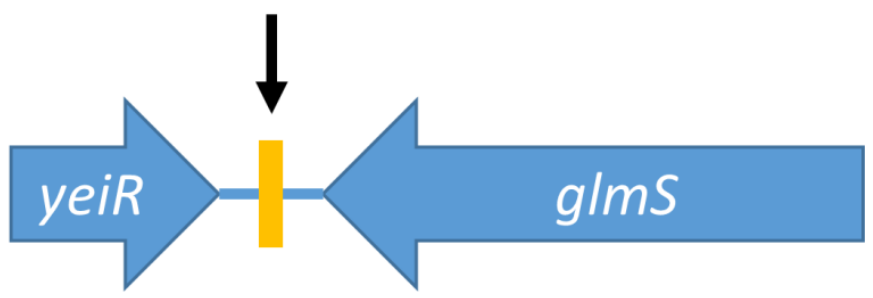

KV6576

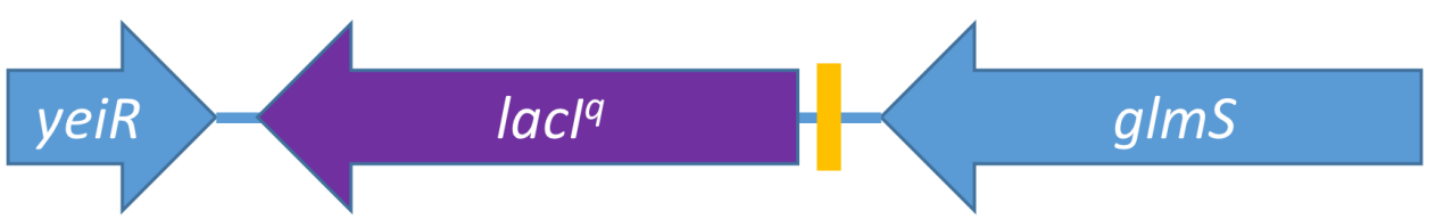

Fig. (2). Construction of a lacI-expressing $\boldsymbol{V}$. fischeri strain. ES114 contains a Tn7 site (yellow rectangle) between yeiR and glmS. KV6576 was engineered to contain the lacI overexpression allele (lacI ${ }^{q}$, purple arrow) near, but not disrupting, the Tn7 site.

Tn7 site, which is adjacent to the site of insertion of the $l a c I^{q}$ gene, still permissive for insertion events? To assess whether the $\operatorname{lacl}^{q}$ allele in KV6576 was functional, we investigated the ability of this strain to impact expression of a lac promoter-controlled gene. Specifically, we introduced into KV6576 a plasmid, pCLD46, that contains the $r s c S$ gene driven by the lac promoter, or pVSV105, the empty vector from which pCLD46 was derived. When pCLD46 is introduced into wild-type $V$. fischeri, RscS protein is made and induces biofilm formation [13]. One biofilm phenotype that can be readily assessed is the formation of wrinkled colonies. We anticipated that, if the lacl $^{\mathrm{q}}$ allele in KV6576 were functional, then LacI would repress expression of $r s c S$, resulting in a strain that either fails to form wrinkled colonies or does so after a delay. Indeed, we found that this strain failed to wrinkle after 24 hours of growth in the absence of IPTG (Fig. 3). Moreover, when we grew the strain in the presence of IPTG, which should inactivate LacI, wrinkled colonies developed with a timing indistinguishable from the control (Fig. 3 and data not shown). These data indicate that functional LacI was made and was responsive to IPTG. We note, however, that the repression of $r s c S$ expression from pCLD46 was not complete: at later times, the strain exhibited a modest wrinkling phenotype in the absence of IPTG (Fig 3). We attribute this result to the inability of LacI expressed from the chromosome to fully repress a promoter present on a multi-copy plasmid.

To verify that the $\operatorname{Tn} 7$ site near the site of $\operatorname{lacl}^{q}$ insertion in KV6576 remained amenable to manipulation, we used pEVS107, a Tn7 delivery vector that targets the Tn7 site [15], to introduce an Erm resistance cassette at that location. pEVS107 contains an Erm resistance cassette within the Tn7 ends and a kanamycin resistance cassette outside. Erythromycin-resistant strains were readily isolated and exhibited sensitivity to kanamycin, as expected when the Tn7 cassette inserts at the Tn7 site (data not shown).

Identification of motility mutants. Our data above indicate that the lacI gene inserted into the chromosome is functional to repress transcription of a lac promotercontrolled gene. However, the question remained, does the lacl ${ }^{\mathrm{q}}$ allele control transcription from the $\mathrm{P}_{\mathrm{A} 1 / 34}$ promoter 

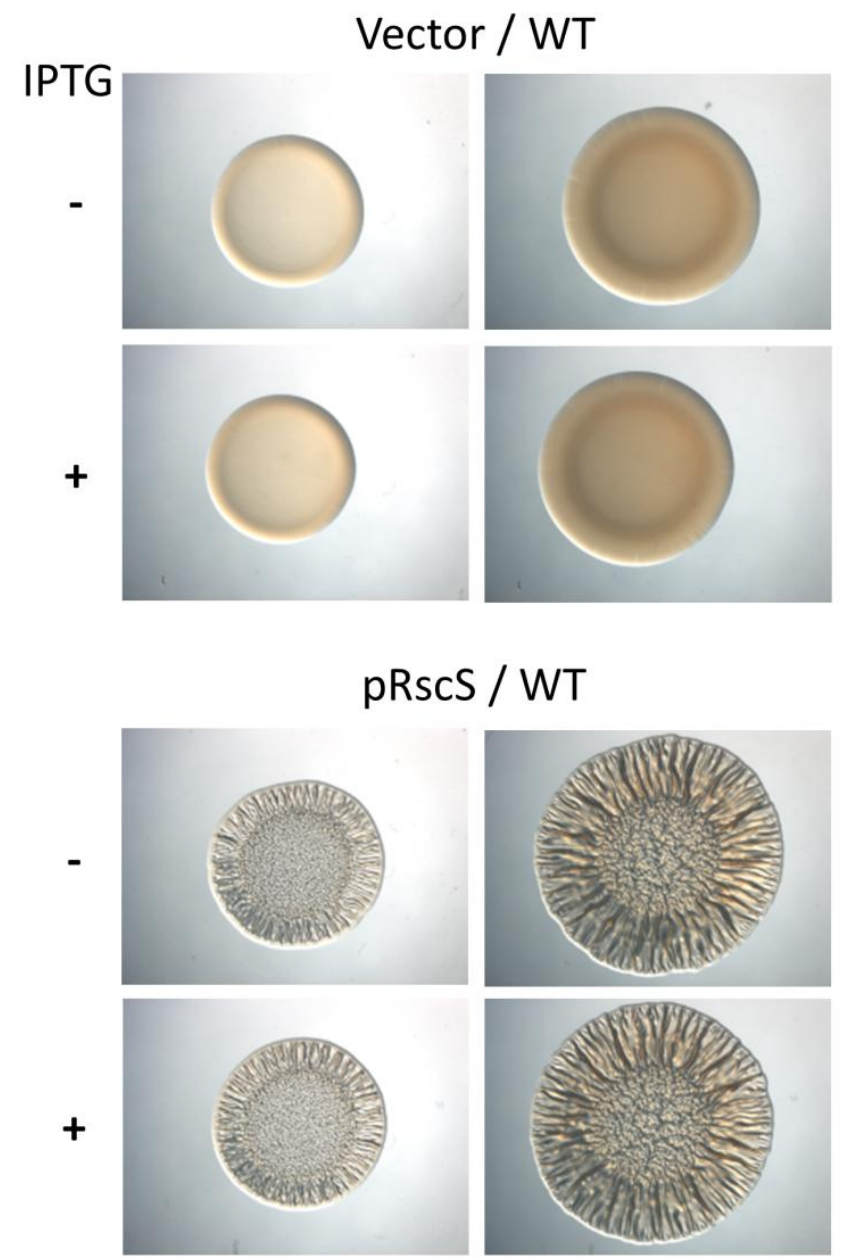

$20 \mathrm{~h}$
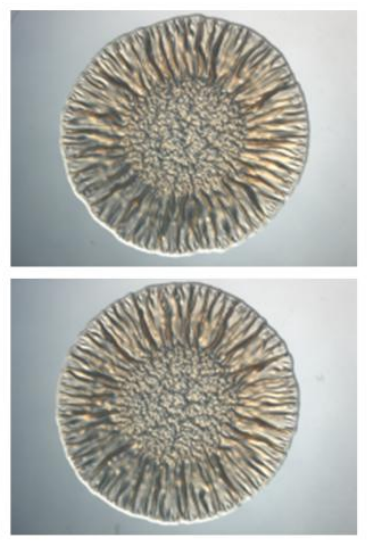

$50 \mathrm{~h}$

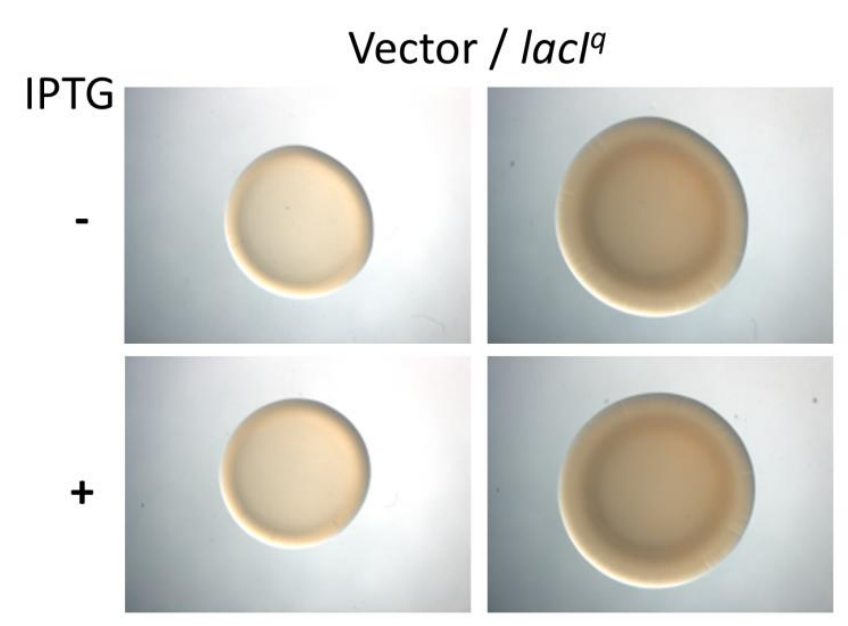

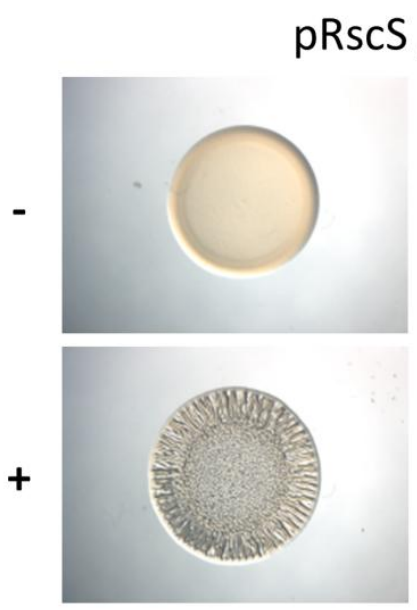

$20 \mathrm{~h}$
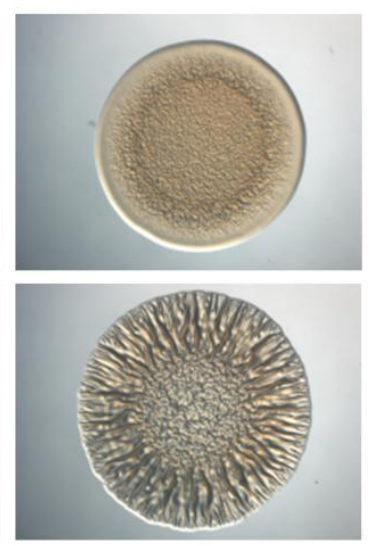

$50 \mathrm{~h}$

Fig. (3). lac promoter-driven biofilm formation by WT and lacI expressing strains. Cultures of wild-type (ES114) and lacI ${ }^{q}$ (KV6576) strains containing either the empty vector pVSV105 or RscS expression plasmid pCLD46 were grown in LBS containing Cm. Aliquots were diluted to an $\mathrm{OD}_{600}$ of 0.2 , spotted onto LBS-Cm medium containing or lacking $1.75 \mathrm{mM}$ IPTG, and incubated at room temperature. Wrinkled colony formation was assessed at 20 and $50 \mathrm{~h}$ post inoculation.

contained within Tn5P? Specifically, we wondered whether we could induce or repress native $V$. fischeri genes in KV6576 containing insertions of Tn5P. To address this question, we chose to evaluate a readily assessable phenotype, motility. V. fischeri contains a number of genes known [35-38] or predicted [39] to impact motility. We hypothesized that $\mathrm{Tn} 5 \mathrm{P}$ insertions upstream of such genes could result in strains with inducible or repressible motility. We thus introduced Tn5P into KV6576 and assessed mutant motility on soft agar plates that contained or lacked IPTG. From a screen of about 2000 mutants, we identified about 20 strains with potential IPTG-dependent motility phenotypes and confirmed the phenotypes of a subset of these mutants. Of these, our focus was drawn to two strains with opposing phenotypes (Fig. 4). One strain, KV7432, had IPTGrepressible motility: it exhibited near wild-type motility in the absence of IPTG but greatly diminished motility in the presence of IPTG (Fig. 4B). In contrast, IPTG did not impact motility of the control strain (Fig. 4A). The second strain of interest, KV7433, had IPTG-inducible motility: it was nonmotile in the absence of IPTG but regained a wild-type motility phenotype in the presence of IPTG (Fig. 4C). Because of their strong yet opposite phenotypes, we chose these two strains for additional characterization.

We first identified the sites of insertion of $\mathrm{Tn} 5 \mathrm{P}$ as described in Materials and Methods. The mutant with IPTGrepressible motility, KV7432, contained an insert within the intergenic region between $V F \_A 0340$ and $V F \_A 0341$, with the promoter of Tn5P oriented toward $V F \_A 0341$ (Fig. 5A). In this orientation, the promoter appears positioned to drive expression of the nearby three-gene operon consisting of $V F_{-} A 0342, V F_{-} A 0343$, and $V F_{-} A 0344$. These three genes are predicted to encode proteins with GGDEF or EAL domains. These domains are found in diguanylate cyclase and phosphodiesterase proteins, which synthesize and degrade, respectively, the second messenger cyclic-di-GMP (c-di-GMP) [40]. High levels of cellular c-di-GMP inhibit motility in a variety of bacteria [41]. Therefore, we hypothesize that IPTG-mediated expression from the transposon promoter increases the levels of c-di-GMP in the cell and thus, inhibits motility. It also remains possible that 


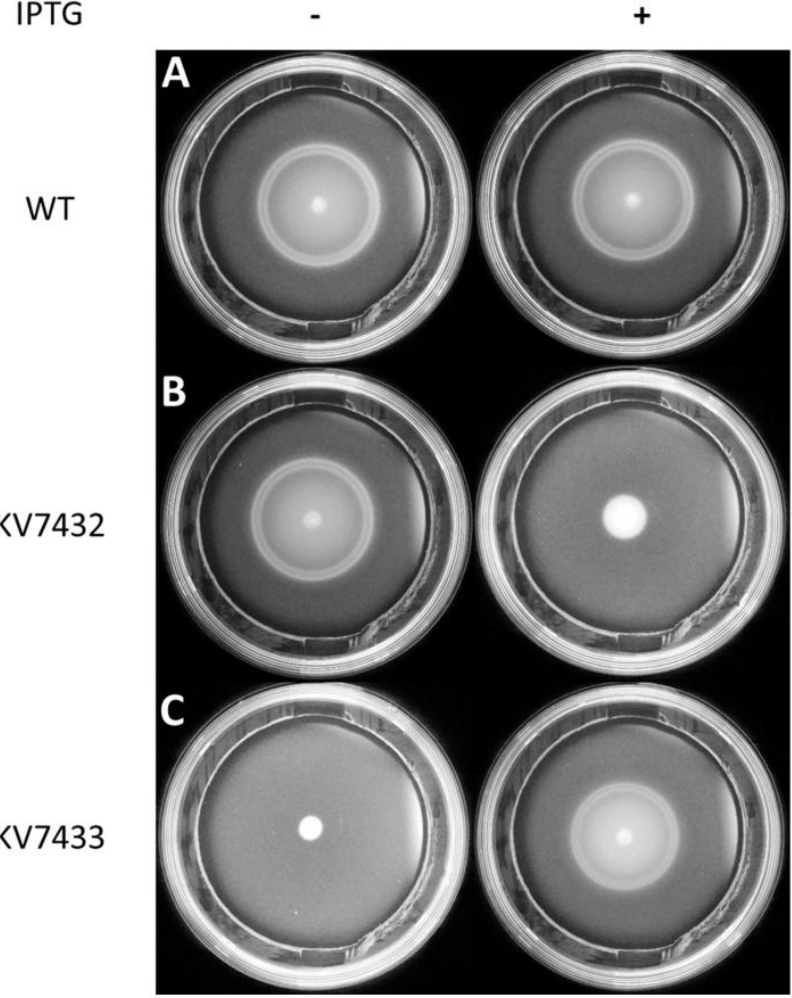

Fig. (4). Migration of mutant strains on soft agar. KV6576 (A), KV7432 (B), and KV7433 (C) were grown overnight in LBS. Cultures were diluted to an $\mathrm{OD}_{600}$ of 0.4 prior to inoculation on TBSW motility medium containing or lacking $1.75 \mathrm{mM}$ IPTG. The images depict migration after $5.5 \mathrm{~h}$ of incubation at $28^{\circ} \mathrm{C}$.

expression from the Tn5P promoter decreases the expression of $V F \_A 0341$, which encodes a hypothetical protein with no conserved domains.

The mutant with IPTG-inducible motility, KV7433, carried the Tn5P insertion within the cheZ gene, with the transposon's promoter oriented with the che operon (Fig. 5B). In the absence of IPTG, the transposon insertion should interrupt transcription of cheZ as well as the downstream che genes which coordinate chemotaxis and are required for motility in $V$. fischeri [38]. Thus, it was not surprising that, in the absence of IPTG, this mutant exhibited a motility defect. Given that the Tn insertion was within the cheZ gene, however, it was unexpected that the addition of IPTG would restore near wild-type motility (Fig. 4). Upon closer investigation of the insertion site, we noted that (1) the Tn is inserted near the beginning of cheZ, and (2) an ATG start codon within the Tn5P transposon end is in frame with the cheZ open reading frame (Fig. 1B and data not shown). Based on these observations, we hypothesize that expression from the transposon's promoter and translation from the ATG within the transposon end results in the production of a hybrid CheZ protein with an altered N-terminus that is functional to promote motility.

Assessment of IPTG Induction. Our previous experiments used a single concentration of IPTG, $1.75 \mathrm{mM}$, to induce transcription from the Tn5P promoter. However, it was unclear whether this high amount of IPTG was necessary to obtain full repression/induction of motility by our strains. Thus, to determine the sensitivity of the Tn5P promoter to IPTG, we assessed the mutants' motility phenotypes in the presence of a range of IPTG concentrations. KV7433 exhibited a dose-dependent increase in motility within a wide range of IPTG concentrations between $3.5 \mu \mathrm{M}$ and $175 \mu \mathrm{M}$ IPTG that was not further increased with additional IPTG (Fig. 6). The other mutant, KV7432, similarly exhibited a dose-dependent change. In this case, the impact on motility required higher IPTG concentrations, above $35 \mu \mathrm{M}$; at the highest amount tested, $1.75 \mathrm{mM}$, motility was not fully repressed (Fig. 6). These data further support our conclusion that expression from the Tn5P promoter is induced by the addition of IPTG. Additionally, because we obtained different ranges of IPTG addition required for a transition from the motile to nonmotile phenotype in the two strains, we conclude that it may

A

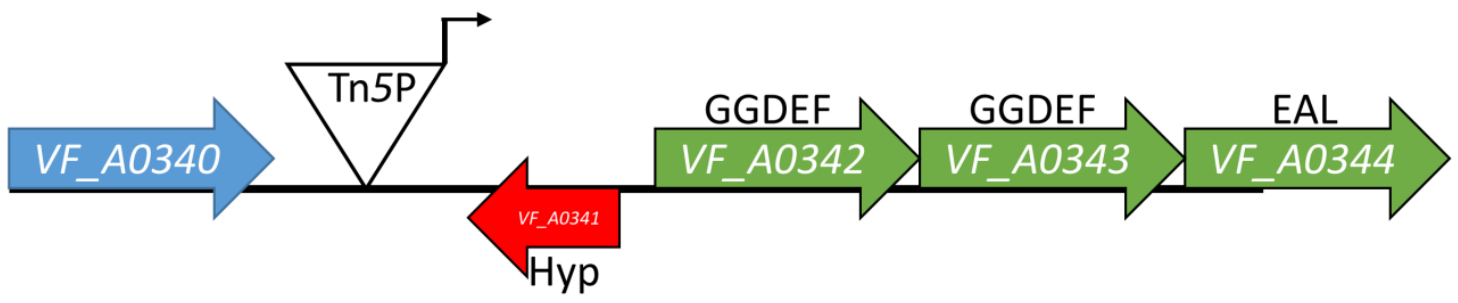

B

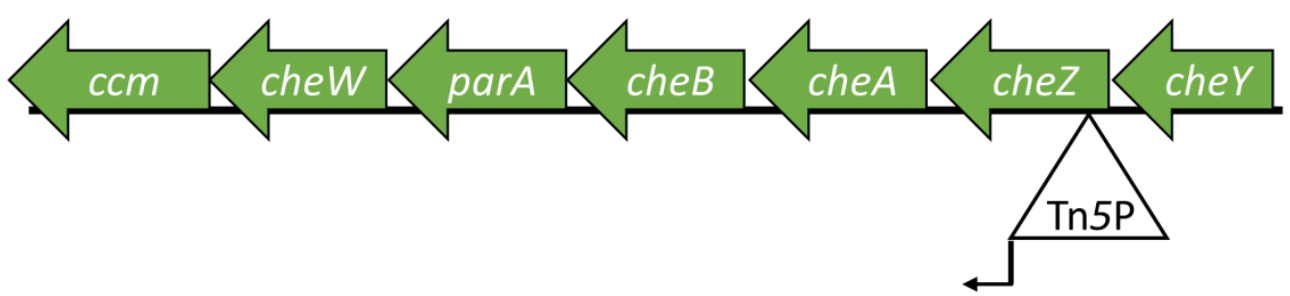

Fig. (5). The location and orientation of the Tn5P insertions in two motility mutants. The insertion in KV7432 is located in the intergenic region between $V F \_A 0340$ and $V F \_A 0341$ with the A1/34 promoter oriented toward $V F \_A 0341$ (A). The insertion in KV7433 is located within the 5' end of cheZ with the A1/34 promoter oriented toward cheA (B). 


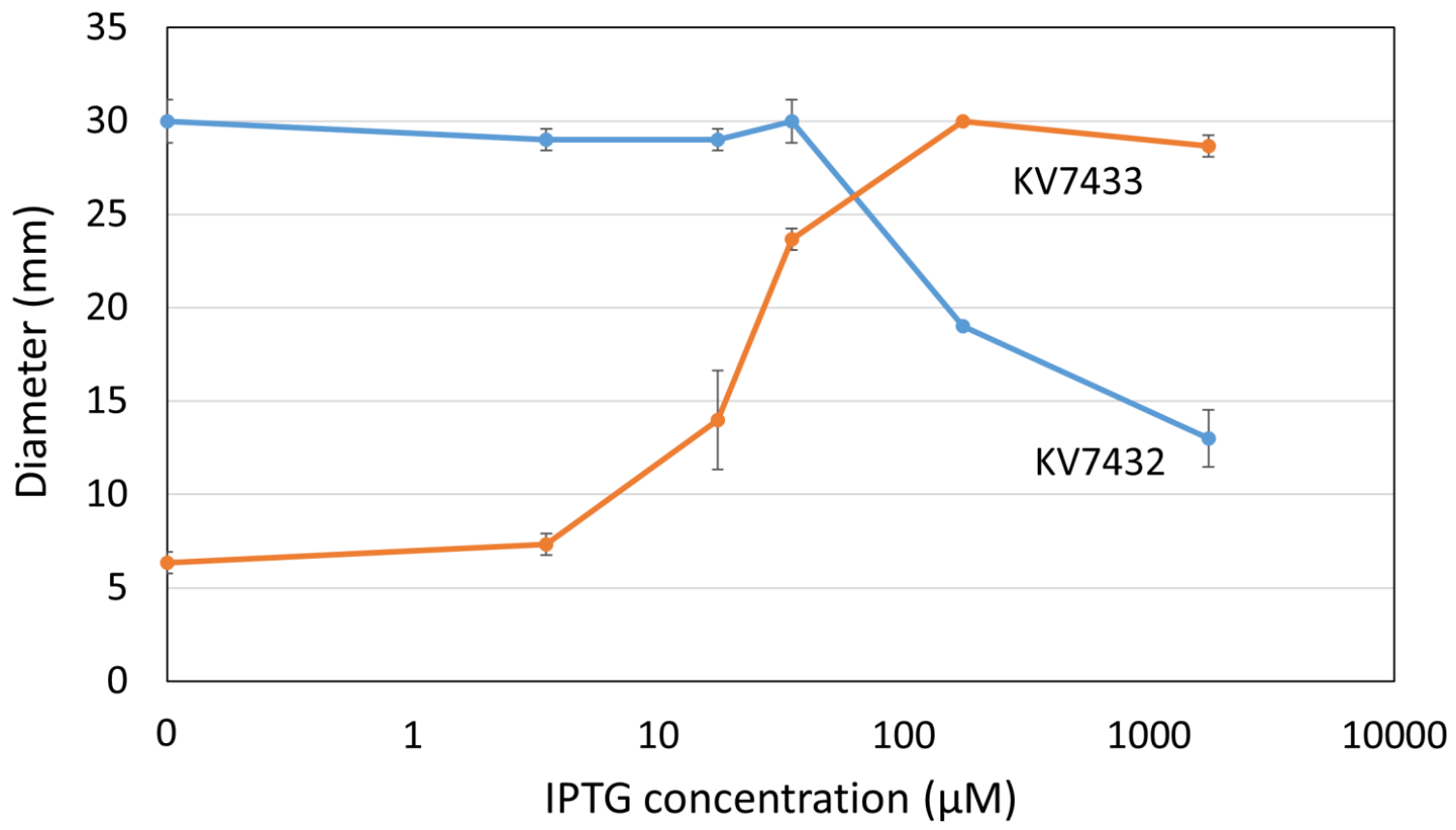

Fig. (6). Dependence of mutant motility phenotypes on IPTG. Motility mutants $\mathrm{KV} 7432$ and $\mathrm{KV} 7433$ were grown at $28^{\circ} \mathrm{C}$ in $\mathrm{TB}-\mathrm{SW}$ motility medium containing the indicated concentrations of IPTG. The average diameter of migration of triplicate samples after 5 hours is shown. Standard deviation is indicated by error bars. Error bars smaller than the plotted points are occluded by the points and not visible.

be necessary to experimentally determine the optimal expression of a particular gene obtained using this experimental set-up $\left(\operatorname{lacl} I^{\mathrm{q}} / \mathrm{Tn} 5 \mathrm{P}\right)$ by titrating the concentration of IPTG in the medium against the phenotype being tested.

\section{CONCLUDING REMARKS}

We have developed a means to randomly insert and control an inducible promoter within the chromosome of $V$. fischeri by (1) generating a mini-Tn5 modified to contain an outward-facing, LacI-repressible promoter and (2) engineering a background strain that expresses LacI to control the promoter. Insertion of the Tn5P within a gene or locus will result in disruption of the gene or locus and has the additional ability to conditionally induce transcription of downstream genes, thus reducing the problem of polarity typically associated with Tn insertions. Finally, our evidence indicates that the Tn5P end may, in some cases, generate and express proteins with N-terminal truncations. Thus, this genetic tool increases the number of phenotypes $V$. fischeri researchers can screen and has the potential to greatly increase our understanding of the physiology of this model organism.

\section{CONFLICT OF INTEREST}

This work was supported by Loyola University Chicago Research funding council and to NIH grant 2R01 GM059690 awarded to KLV.

\section{ACKNOWLEDGEMENTS}

We thank Eric Stabb for suggesting the use of the A1/34 promoter for this study, and members of the Visick lab for critically reading this manuscript.

\section{REFERENCES}

[1] Bose JL, Rosenberg CS, Stabb EV. Effects of luxCDABEG induction in Vibrio fischeri: enhancement of symbiotic colonization and conditional attenuation of growth in culture. Arch Microbiol 2008; 190: 169-83.

[2] Septer AN, Stabb EV. Coordination of the arc regulatory system and pheromone-mediated positive feedback in controlling the Vibrio fischeri lux operon. PLoS One 2012; 7: e49590.

[3] Verma SC, Miyashiro T. Quorum sensing in the squid-Vibrio symbiosis. Int J Mol Sci 2013; 14: 16386-401.

[4] Yildiz FH, Visick KL. Vibrio biofilms: so much the same yet so different. Trends Microbiol 2009; 17: 109-18.

[5] Visick KL. An intricate network of regulators controls biofilm formation and colonization by Vibrio fischeri. Mol Microbiol 2009; 74: 782-9.

[6] Norsworthy AN, Visick KL. Gimme shelter: how Vibrio fischeri successfully navigates an animal's multiple environments. Front Microbiol 2013; 4: 356.

[7] Stabb E, Visick KL. Vibrio fischeri: A Bioluminescent Light-Organ Symbiont of the Bobtail Squid Euprymna scolopes. In: Rosenberg E, DeLong EF, Stackebrandt E, Lory S, Thompson F, Eds. The Prokaryotes - Prokaryotic Biology and Symbiotic Associations. $4^{\text {th }}$ ed, USA: Springer. 2013; pp. 497-532.

[8] Robertson LA, Figge MJ, Dunlap PV. Beijerinck and the bioluminescent bacteria: microbiological experiments in the late 19th and early 20th centuries. FEMS Microbiol Ecol 2011; 75: 185-94.

[9] Dunlap PV, Kuo A. Cell density-dependent modulation of the Vibrio fischeri luminescence system in the absence of autoinducer and LuxR protein. J Bacteriol 1992; 174: 2440-8.

[10] Graf J, Dunlap PV, Ruby EG. Effect of transposon-induced motility mutations on colonization of the host light organ by Vibrio fischeri. J Bacteriol 1994; 176: 6986-91.

[11] Visick KL, Ruby EG. New Genetic Tools for use in the Marine Bioluminescent Bacterium Vibrio fischeri. In: Hastings JW, Kricka LJ, Stanley PE, Eds. Bioluminescence and Chemiluminescence. NY: John Wiley and Sons, 1997; pp. 119-22.

[12] Dunn AK, Millikan DS, Adin DM, Bose JL, Stabb EV. New rfpand pES213-derived tools for analyzing symbiotic Vibrio fischeri reveal patterns of infection and lux expression in situ. Appl Environ Microbiol 2006; 72: 802-10. 
[13] Hussa EA, Darnell CL, Visick KL. RscS functions upstream of SypG to control the syp locus and biofilm formation in Vibrio fischeri. J Bacteriol 2008; 190: 4576-83.

[14] Simon R, Priefer U, Puhler A. A broad host range mobilization system for In vivo genetic engineering: transposon mutagenesis in Gram negative bacteria. Nat Biotechnol 1983; 1: 784-91.

[15] McCann J, Stabb EV, Millikan DS, Ruby EG. Population dynamics of Vibrio fischeri during infection of Euprymna scolopes. Appl Environ Microbiol 2003; 69: 5928-34.

[16] DeLoney-Marino CR, Visick KL. Role for cheR of Vibrio fischeri in the Vibrio-squid symbiosis. Can J Microbiol 2012; 58: 29-38.

[17] Ray VA, Visick KL. LuxU connects quorum sensing to biofilm formation in Vibrio fischeri. Mol Microbiol 2012; 86(4): 954-70.

[18] Morris AR, Visick KL. Inhibition of SypG-induced biofilms and host colonization by the negative regulator SypE in Vibrio fischeri. PLoS One 2013; 8: e60076.

[19] Ray VA, Eddy JL, Hussa EA, Misale M, Visick KL. The syp enhancer sequence plays a key role in transcriptional activation by the $\sigma^{54}$-dependent response regulator $\operatorname{SypG}$ and in biofilm formation and host colonization by Vibrio fischeri. J Bacteriol 2013; 195: 5402-12.

[20] Ruby EG, Urbanowski M, Campbell J, et al. Complete genome sequence of Vibrio fischeri: a symbiotic bacterium with pathogenic congeners. Proc Natl Acad Sci U S A 2005; 102: 3004-9.

[21] Le Roux F, Binesse J, Saulnier D, Mazel D. Construction of a Vibrio splendidus mutant lacking the metalloprotease gene vsm by use of a novel counterselectable suicide vector. Appl Environ Microbiol 2007; 73: 777-84.

[22] Shibata S, Visick KL. Sensor kinase RscS induces the production of antigenically distinct outer membrane vesicles that depend on the symbiosis polysaccharide locus in Vibrio fischeri. J Bacteriol 2012; 194: 185-94.

[23] Aeckersberg F, Lupp C, Feliciano B, Ruby EG. Vibrio fischeri outer membrane protein OmpU plays a role in normal symbiotic colonization. J Bacteriol 2001; 183: 6590-7.

[24] Visick KL, Quirke KP, McEwen SM. Arabinose induces pellicle formation by Vibrio fischeri. Appl Environ Microbiol 2013; 79 : 2069-80.

[25] Stabb EV, Reich KA, Ruby EG. Vibrio fischeri genes hvnA and $h v n B$ encode secreted NAD(+)-glycohydrolases. J Bacteriol 2001; 183: 309-17.

[26] DeLoney-Marino CR, Wolfe AJ, Visick KL. Chemoattraction of Vibrio fischeri to serine, nucleosides, and $\mathrm{N}$-acetylneuraminic acid, a component of squid light-organ mucus. Appl Environ Microbiol 2003; 69: 7527-30.

[27] Herrero M, de Lorenzo V, Timmis KN. Transposon vectors containing non-antibiotic resistance selection markers for cloning and stable chromosomal insertion of foreign genes in gramnegative bacteria. J Bacteriol 1990; 172: 6557-67.

[28] Bertani G. Studies on lysogenesis. I. The mode of phage liberation by lysogenic Escherichia coli. J Bacteriol 1951; 62: 293-300.

[29] Ho SN, Hunt HD, Horton RM, Pullen JK, Pease LR. Site-directed mutagenesis by overlap extension using the polymerase chain reaction. Gene 1989; 77: 51-9.

[30] Lanzer M, Bujard H. Promoters largely determine the efficiency of repressor action. Proc Natl Acad Sci U S A 1988; 85: 8973-77.

[31] Lyell NL, Dunn AK, Bose JL, Vescovi SL, Stabb EV. Effective mutagenesis of Vibrio fischeri by using hyperactive mini-Tn5 derivatives. Appl Environ Microbiol 2008; 74: 7059-63.

[32] Stabb EV, Ruby EG. RP4-based plasmids for conjugation between Escherichia coli and members of the Vibrionaceae. Methods Enzymol 2002; 358: 413-26.

[33] Lichtenstein C, Brenner S. Unique insertion site of $\operatorname{Tn} 7$ in the $E$. coli chromosome. Nature 1982; 297: 601-3.

[34] Morris AR, Darnell CL, Visick KL. Inactivation of a novel response regulator is necessary for biofilm formation and host colonization by Vibrio fischeri. Mol Microbiol 2011; 82: 114-30.

[35] Millikan DS, Ruby EG. FlrA, a $\sigma^{54}$-dependent transcriptional activator in Vibrio fischeri, is required for motility and symbiotic light-organ colonization. J Bacteriol 2003; 185: 3547-57.

[36] Millikan DS, Ruby EG. Vibrio fischeri flagellin A is essential for normal motility and for symbiotic competence during initial squid light organ colonization. J Bacteriol 2004; 186: 4315-25.

[37] Wolfe AJ, Millikan DS, Campbell JM, Visick KL. Vibrio fischeri $\sigma^{54}$ controls motility, biofilm formation, luminescence, and colonization. Appl Environ Microbiol 2004; 70: 2520-4.

[38] Brennan CA, Mandel MJ, Gyllborg MC, Thomasgard KA, Ruby EG. Genetic determinants of swimming motility in the squid lightorgan symbiont Vibrio fischeri. Microbiologyopen 2013; 2: 576-94.

[39] Wolfe AJ, Visick KL. Roles of Diguanylate Cyclases and Phosphodiesterases in Motility and Biofilm Formation in Vibrio fischeri. In: Wolfe AJ, Visick KL, Eds. The Second Messenger, Cyclic-Di-GMP. Washington, DC, ASM Press 2010; pp. 186-200.

[40] Wolfe AJ, Visick KL. The Second Messenger Cyclic Di-GMP. Washington, DC: ASM Press 2010.

[41] Romling U, Galperin MY, Gomelsky M. Cyclic di-GMP: the first 25 years of a universal bacterial second messenger. Microbiol Mol Biol Rev 2013; 77: 1-52.

[42] Boettcher KJ, Ruby EG. Depressed light emission by symbiotic Vibrio fischeri of the sepiolid squid Euprymna scolopes. J Bacteriol 1990; 172: 3701-6.

[43] Kitagawa M, Ara T, Arifuzzaman M, et al. Complete set of ORF clones of Escherichia coli ASKA library (a complete set of E. coli K-12 ORF archive): unique resources for biological research. DNA Res 2005; 12: 291-9.

(C) Ondrey and Visick; Licensee Bentham Open.

This is an open access article licensed under the terms of the Creative Commons Attribution Non-Commercial License (http://creativecommons.org/licenses/by-nc/3.0/) which permits unrestricted, non-commercial use, distribution and reproduction in any medium, provided the work is properly cited. 